\title{
Neurolymphomatosis in the Cauda Equina Diagnosed by an Open Biopsy
}

\author{
Ryo Sasaki ${ }^{1}$, Yasuyuki Ohta ${ }^{1}$, Yuto Yamada ${ }^{1}$, Koh Tadokoro ${ }^{1}$, Yoshiaki Takahashi ${ }^{1}$, \\ Kota Sato ${ }^{1}$, Jingwei Shang ${ }^{1}$, Mami Takemoto ${ }^{1}$, Nozomi Hishikawa ${ }^{1}$, Toru Yamashita ${ }^{1}$, \\ Takao Yasuhara $^{2}$, Isao Date ${ }^{2}$, Shuntaro Ikegawa ${ }^{3}$, Nobuharu Fujii ${ }^{3}$ and Koji Abe ${ }^{1}$
}

\begin{abstract}
:
Neurolymphomatosis is a rare form of extranodal malignant lymphoma defined as the infiltration of malignant lymphocytes into the central or peripheral nerve. We herein report a case of neurolymphomatosis in the cauda equina diagnosed by an open surgical biopsy. He presented with muscle weakness, atrophy, numbness and hypoesthesia in the bilateral lower extremities with the accumulation of ${ }^{18}$ fluoro-2-deoxyglucose (FDG) in the bilateral cauda equina. Cerebrospinal fluid cytology (three times) and flow cytometry (two times) and biopsies of the left sural nerve, bone marrow, paranasal sinus and left testis were all negative for malignancy, so finally we performed a surgical open biopsy of the cauda equina by laminectomy and diagnosed him with diffuse large B-cell lymphoma in the cauda equina. He was successfully treated with the disappearance of the FDG accumulation for a long time. The present case suggested that an early open biopsy of the cauda equina may be considered for cases of suspected neurolymphomatosis in the cauda equina for a good outcome.
\end{abstract}

Key words: neurolymphomatosis, cauda equina, spinal biopsy, B-cell lymphoma

(Intern Med 57: 3463-3465, 2018)

(DOI: 10.2169/internalmedicine.1049-18)

\section{Introduction}

Neurolymphomatosis is a very rare form of extranodal malignant lymphoma defined as the infiltration of malignant lymphocytes into the central or peripheral nerve. Neurolymphomatosis in the spinal cord or cauda equina accounts for less than $1 \%$ of all cases of neurolymphomatosis, and about $90 \%$ of cases of neurolymphomatosis are diffuse large Bcell lymphoma (DLBCL) (1). The early diagnosis and treatment result in a good outcome and increase the survival rate (2). Gadolinium (Gd)-enhanced magnetic resonance imaging (MRI) and ${ }^{18}$ fluoro-2-deoxyglucose positron emission tomography (FDG-PET) have proven useful for detecting malignancy, but histopathological studies are essential for the diagnosis of neurolymphomatosis. However, the sensitivity of a peripheral nerve biopsy or CSF cytology is low.
Therefore, a surgical open biopsy of the cauda equine may be considered for the diagnosis of neurolymphomatosis.

We herein report a rare case of neurolymphomatosis in the cauda equina that was ultimately diagnosed by an open biopsy and successfully treated with the disappearance of the FDG accumulation for a long time.

\section{Case Report}

A 62-year-old man gradually developed muscle weakness and numbness in the bilateral lower extremities (LEs) and became unable to walk without human support after 16 months, at which point he was admitted to our hospital.

On a neurological examination, he showed muscle weakness, atrophy and fasciculations in the bilateral LEs, and numbness and hypoesthesia in the bilateral distal LEs with absent bilateral patellar and Achilles reflexes. However, he

\footnotetext{
${ }^{1}$ Department of Neurology, Graduate School of Medicine, Dentistry and Pharmaceutical Sciences, Okayama University, Japan, ${ }^{2}$ Department of Neurological Surgery, Graduate School of Medicine, Dentistry and Pharmaceutical Sciences, Okayama University, Japan and ${ }^{3}$ Department of Hematology and Oncology, Graduate School of Medicine, Dentistry and Pharmaceutical Sciences, Okayama University, Japan Received: February 20, 2018; Accepted: May 1, 2018; Advance Publication by J-STAGE: August 10, 2018 Correspondence to Dr. Ryo Sasaki, ryosasaki@okayama-u.ac.jp
} 

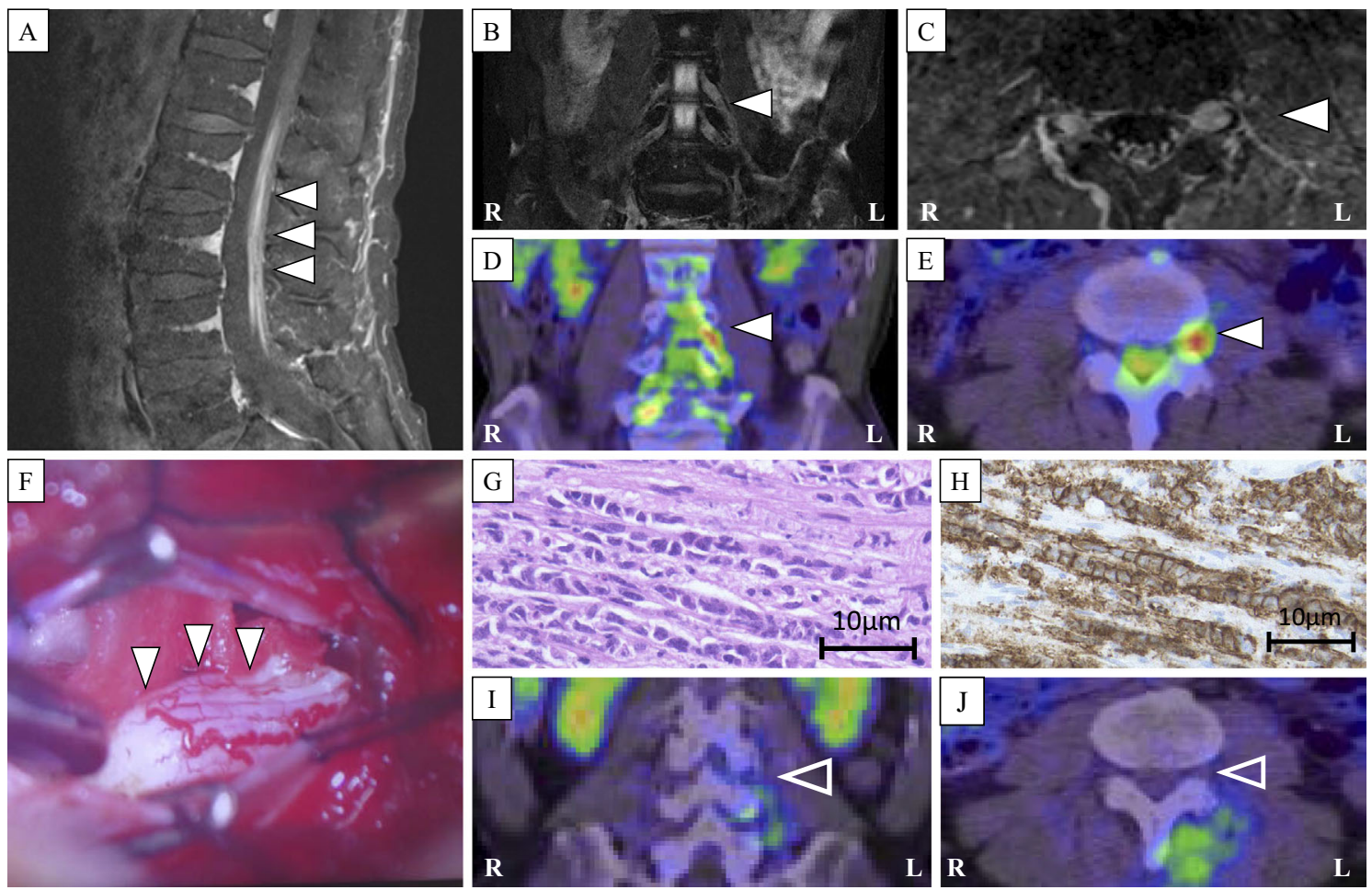

Figure. (A-C) Gd-enhanced T1-weighted MRI showing contrast enhancement in the cauda equina (A, arrowheads) and thickening of the nerve roots (right<left) (B, C, arrowheads). (D, E) FDG-PET showing the accumulation of FDG in the cauda equina (D, arrowhead) and nerve roots ( $E$, arrowhead). (F) Thickening of the cauda equina (arrowheads) was confirmed by an open biopsy. (G, H) A pathological study shows the diffuse proliferation of large round cells in the nerve tissue of the cauda equina (G, Hematoxylin and Eosin staining); the cells were CD20-positive (H). (I, J) The disappearance of the FDG accumulation on FDG-PET after treatment (arrowheads).

showed no other remarkable findings of the cranial nerve, cerebellar or autonomic system, such as urinary disturbance.

A motor nerve conduction study showed a low amplitude $(85.0 \mu \mathrm{V}$, normal range $7.3-21.0 \mathrm{mV}$ ) in the right tibial nerve. A sensory nerve conduction study showed no evoked potentials of the bilateral sural nerves but normal evoked potentials of the bilateral median and ulnar nerves. A biochemical study showed a normal level of creatine phosphokinase (CPK; $134 \mathrm{U} / \mathrm{L}$, normal 59-248 U/L), lactate dehydrogenase (LDH) (147 U/L, normal 124-222 U/L) and tumor markers, including soluble interleukin-2 (IL-2) receptor (403 $\mathrm{U} / \mathrm{mL}$, normal $122-496 \mathrm{U} / \mathrm{mL}$ ). A cerebral spinal fluid study showed pleocytosis $(41 / \mu \mathrm{L}$, monocyte dominant, normal $\leq 3 /$ $\mu \mathrm{L})$ and an elevated protein $(325 \mathrm{mg} / \mathrm{dL}$, normal $8-40 \mathrm{mg} /$ $\mathrm{dL})$ and $\mathrm{IgG}$ index $(0.91$, normal $\leq 0.60)$.

Gadolinium (Gd)-enhanced T1-weighted magnetic resonance imaging (MRI) showed thickening of the nerve roots (right<left) with contrast enhancement in the cauda equina (Figure A-C, arrowheads). FDG-PET showed the accumulation of FDG in the cauda equina and left nerve roots (Figure $\mathrm{D}$ and $\mathrm{E}$, arrowheads) corresponding with the enhanced lesions of MRI (Figure B and C, arrowheads), as well as in the paranasal sinus and left testis (data not shown).

Based on the above findings, malignant tumors, including hematological malignancy, were suspected. However, cere- brospinal fluid (CSF) cytology three times, CSF flow cytometry twice and left sural nerve and bone marrow biopsies showed no evidence of malignancy. We therefore performed paranasal sinus and left testis biopsies for possible lesions, but they also showed no evidence of malignancy.

Finally, we performed a surgical open biopsy of the cauda equina by L3 laminectomy at 40 days after admission. We performed posterior laminectomy of the L3 vertebrae based on the MRI and FDG-PET findings and removed the macroscopically thickening part of the cauda equina (Figure F, arrowheads) while monitoring the motor evoked potential (MEP) and bulbocavernous reflex (BCR). There was no evident deterioration of the muscle, sensory disturbance or urinary disturbance after the biopsy. A pathological study found diffuse proliferation of large round cells in the nerve tissue of the cauda equina (Figure G). An immunohistological examination showed positive cluster of differentiation 20 (CD20) staining of large round cells (Figure H). We therefore diagnosed the patient with diffuse large B-cell lymphoma (DLBCL) in the cauda equina.

Six intravenous administrations of high-dose methotrexate $\left(3,500 \mathrm{mg} / \mathrm{m}^{2}\right.$ of body-surface area) and rituximab $(375 \mathrm{mg} /$ $\mathrm{m}^{2}$ of body-surface area) combined with irradiation therapy at the lumbar spine ( 2 Gy $\times 15$ times) greatly improved his muscle weakness and atrophy of the bilateral LEs, and he 
became able to walk with a cane. We confirmed the disappearance of the previous accumulation on FDG-PET (Figure $\mathrm{I}$ and $\mathrm{J}$, arrowheads). He was discharged three months after the treatment, and he was still able to walk without recurrence of the FDG accumulation at eight months after discharge (data not shown).

\section{Discussion}

The present case showed primary neurolymphomatosis in the cauda equina that was finally diagnosed by an open biopsy. He showed muscle weakness, atrophy, numbness and hypoesthesia in the bilateral distal LEs with thickening nerve roots $(\mathrm{R}<\mathrm{L})$ on Gd-enhanced MRI (Figure A-C, arrowheads). Although the accumulation of FDG was found in the cauda equina, left nerve roots (Figure D and E, arrowheads), paranasal sinus and left testis, standard examinations, such as CSF cytology (three times) and biopsies of left sural nerve, bone marrow, paranasal sinus and left testis, were all negative for malignancy. Thus, a surgical open biopsy of the cauda equina (Figure F, arrowheads) finally provided the diagnosis of DLBCL. After six rounds of chemotherapy with irradiation therapy, the symptoms successfully improved, and the FDG accumulation disappeared (Figure I and J, arrowheads).

Neurolymphomatosis is an extranodal lymphoma in the central or peripheral nervous system and is rare in the spinal cord or cauda equina, accounting for less than $1 \%$ of all neurolymphomatosis (3). About $90 \%$ of cases of neurolymphomatosis are DLBCL (1). Similar to previous cases of neurolymphomatosis in the cauda equina (4), the present case also showed asymmetrical polyneuropathy without pain (Figure B-E). Gd-enhanced MRI and FDG-PET are useful for detecting small or insidious malignancies, but the diagnosis is still difficult in some cases (5). Indeed, biopsies of the paranasal sinus and left testis failed to show evidence of malignancy in the present case.

Limitations of MRI, FDG-PET and CSF cytology in cases of neurolymphomatosis have been reported $(6,7)$. A previous report showed that CSF cytology combined with flow cytometry increased the sensitivity of findings for malignancy (8). However, CSF cytology three times and flow cytometry twice revealed no evidence of malignancy in the present case. Thus, the diagnosis of DLBCL was confirmed pathologically in the present case by an open biopsy at 40 days after the admission, resulting in a good outcome and increased survival rates (2).

We herein report a rare case of neurolymphomatosis in the cauda equina diagnosed by an open biopsy, in which FDG-PET showed the disappearance of the FDG accumulation for a long time after treatment. The present case suggests that an early open biopsy of the cauda equina may be useful in the cases of suspected neurolymphomatosis in the cauda equina. Furthermore, FDG-PET was useful for identifying the target of a biopsy and confirming a lack of recurrence of malignancy.

The authors state that they have no Conflict of Interest (COI).

\section{Financial Support}

This work was supported in part by Grants-in-Aid for Scientific Research (B) 25293202, (C) 15K15527 and Young Research 15K21181, and by Grants-in-Aid from the Research Committees (Mizusawa H, Nakashima K, Nishizawa M, Sasaki H, and Aoki M) from the Ministry of Health, Labour and Welfare of Japan.

\section{Acknowledgement}

We appreciate the cooperation of the patient.

\section{References}

1. Iwamoto FM, DeAngelis LM. An update on primary central nervous system lymphoma. Hematol Oncol Clin North Am 20: 12671285, 2006.

2. Yamada S, Tanimoto A, Nabeshima A, et al. Diffuse large B-cell lymphoma presenting with neurolymphomatosis and intravascular lymphoma: a unique autopsy case with diverse neurological symptoms. Diagn Pathol 13; 7: 94, 2012.

3. Baehring JM, Damek D, Martin EC, Betensky RA, Hochberg FH. Neurolymphomatosis. Neuro Oncol 5: 104-115, 2003.

4. Ramirez-Zamora A, Morales-Vidal S, Chawla J, Biller J. Autopsy proven peripheral nervous system neurolymphomatosis despite negative bilateral sural nerve biopsy. Front Neurol 4: 197, 2013.

5. Yazawa S, Ohi T, Shiomi K, Takashima N, Kyoraku I, Nakazato M. Brachial plexus neurolymphomatosis: a discrepancy between electrophysiological and radiological findings. Intern Med 46: 533534, 2007.

6. van den Bent MJ, de Bruin HG, Bos GM, Brutel de la Riviere G, Sillevis Smitt PA. Negative sural nerve biopsy in neurolymphomatosis. J Neurol 246: 1159-1163, 1999.

7. Bokstein F, Goor O, Shihman B, et al. Assessment of neurolymphomatosis by brachial plexus biopsy and PET/CT. Report of a case. J Neurooncol 72: 163-167, 2005.

8. Scott BJ, Douglas VC, Tihan T, Rubenstein JL, Josephson SA. A systematic approach to the diagnosis of suspected central nervous system lymphoma. JAMA Neurol 70: 311-319, 2013.

The Internal Medicine is an Open Access journal distributed under the Creative Commons Attribution-NonCommercial-NoDerivatives 4.0 International License. To view the details of this license, please visit (https://creativecommons.org/licenses/ by-nc-nd/4.0/).

(C) 2018 The Japanese Society of Internal Medicine Intern Med 57: 3463-3465, 2018 